\title{
Los procedimientos de emergencia en la contratación pública*
}

Juan Francisco Díaz**

\section{RESUMEN}

El 16 de abril de 2016, cerca de las 19h00, tuvo lugar un terremoto frente a las costas del Ecuador. Al día siguiente, para Manabí, Esmeraldas y el resto del país, todo fue distinto. Desde el ámbito de la contratación pública, mucho trecho tuvo que transcurrir para que se pueda reconstruir lo devastado. A más de un año transcurrido, se analiza cómo se procedió al amparo los procedimientos de contratación por emergencia; las actuaciones de la administración pública y sus funcionarios dentro de un estado de excepción, para finalmente verificar su reparación integral. PaLABRAS CLAVE: emergencia, terremoto, contratación pública, estado de excepción, fuerza mayor.

\section{ABSTRACT}

On Saturday, April 16, 2016, around 19h00, the earthquake started in front of the Ecuador coasts. The next day was different for Esmeraldas, Manabí and the whole country. Talking about public procurement, takes too long to rebuild the devastated areas. A year later, was analyzed how to proceeded in the emergency; the actions of the public administration and its officials in a state of emergency and verify the integral reparation.

KEYWORDS: emergency, earthquake, procurement, state of emergency, greater strength.

\section{INTRODUCCIÓN}

T as contrataciones por emergencia son aquellas que permiten suplir de manera inmediata las necesidades de bienes, obras o servicios, sin llevar a cabo un pro-

* $\quad$ Este artículo se terminó de escribir en julio de 2017.

** Abogado en libre ejercicio, y docente contratado de la Universidad Andina Simón Bolívar, Sede Ecuador. 
cedimiento de contratación convencional, siempre y cuando medie un evento no predecible y probado.

Ha transcurrido más de un año desde el lamentable embate de la naturaleza que sufrió el Ecuador -en especial las provincias de Manabí y Esmeraldas-al soportar un movimiento telúrico de 7,8 grados en la escala de Richter. A pesar de los esfuerzos, se lamentó el fallecimiento de 663 personas, hubo nueve desaparecidos, 6.274 personas heridas, fueron entregados 737.787 kits de alimentos, 28.827 personas se albergaron, y 113 personas fueron rescatadas con vida entre los escombros. ${ }^{1}$

A los datos anteriores se suman las pérdidas materiales: según la Secretaría Nacional de Planificación y Desarrollo (SENPLADES), 6.622 viviendas y 71 kilómetros de vías fueron destruidas por el terremoto. Como información adicional relevante es necesario particularizar que, según el Instituto Nacional de Estadística y Censos (INEC), el 42,3\% de la población de los diez cantones más afectados en Manabí y Esmeraldas, a raíz del terremoto del 16 de abril, vivía bajo la línea de pobreza por consumo, y el $74,4 \%$ no contaba con servicios básicos. En total hubo 1'015.613 ciudadanos expuestos al terremoto ${ }^{2}$.

Ante esta dura realidad a raíz de la catástrofe es momento de reconstruir y de rehacer lo devastado. El cómo, cuándo y dónde de la reconstrucción dependió, depende y dependerá de manera importante, de cuán efectivos y eficientes sean los procedimientos de contratación.

\section{ACTOS ADMINISTRATIVOS RELEVANTES}

Las emergencias, al ser actos administrativos plenamente discrecionales de la administración pública, entendiéndose discrecional como "la elección de una entre varias opciones igualmente válidas, dentro de los límites de la potestad y de la competencia del órgano", ${ }^{3}$ y al relacionar de manera importante la práctica y el dogma en la contratación administrativa -sin afectar la legalidad, aunque existen casos- es im-

1. Revisar Informe de situación No. 71 de la Secretaría de Gestión de Riesgos de 19 de mayo de 2016. Disponible en 〈http://www.gestionderiesgos.gob.ec/wp-content/uploads/downloads/2016/05/Informede-Situaci\%C3\%B3n-69-\%E2\%80\%93-19052016-\%E2\%80\%93-12H30.pdf . Consulta: 6 de marzo de 2017.

2. "6.622 viviendas y 71 kilómetros de vías fueron destruidas por el terremoto", El Telégrafo, 23 de abril de 2016. Disponible en 〈http://www.eltelegrafo.com.ec/noticias/ecuador/3/6-622-viviendas-fueron-destruidas-por-el-terremoto . Consulta: 6 de marzo de 2017.

3. Ecuador, Reglamento para el Control de la Discrecionalidad, Decreto Ejecutivo 3179, Registro Oficial 686 (Quito, 18 de octubre de 2002), art. 2. 
portante examinar no solamente la generalidad, sino es ineludible observar y analizar las acciones burocráticas posteriores al evento natural referido.

Comencemos por el principio. El 16 de abril de 2016, a las 18h58, sucedió el terremoto, cuyo epicentro fue cerca de las costas del Ecuador frente a las poblaciones de Pedernales y Cojimíes, provincia de Manabí. Para ese entonces, el Presidente de la República se encontraba fuera del país en visita oficial, por lo que al día siguiente del evento natural, desde Roma, expide el primer decreto ejecutivo relacionado con esta catástrofe, signándole el No. 1001, donde se declara el estado de excepción por sesenta días en las provincias de Esmeraldas, Manabí, Santa Elena, Santo Domingo de los Tsáchilas, Los Ríos y Guayas.

Posteriormente se suscribe un alcance mediante decreto No. 1002 de 18 de abril de 2016, que indica que la movilización -que en el decreto anterior estaba dado únicamente para las provincias antes señaladas- sea a nivel nacional; además se dispone las requisiciones necesarias sin olvidar el cumplimiento de las disposiciones legales vigentes.

El 18 de abril el Servicio Nacional de Contratación Pública (SERCOP) expide un oficio circular No. SERCOP-DG-2016-0284-OF que recuerda a las autoridades de las distintas entidades públicas que existe un procedimiento especial denominado "de emergencia", y, en un alcance al citado oficio, ahora con No. SERCOP-DG-20160289-OF de 21 de abril, indica -entre otras cosas- que no se puede alegar impedimentos administrativos o burocráticos a fin de llevar a cabo las contrataciones necesarias; además indican que la resolución declaratoria de emergencia y otros documentos se publiquen posteriormente, finalmente se ponen a disposición formatos de documentos.

El mismo 18 de abril el SERCOP expide la resolución No. SERCOP-RE-048-2016, en donde el director general subrogante resuelve en lo principal declarar en emergencia el portal, e indica que las dependencias deben utilizar el procedimiento de emergencia, de manera directa sin concurso previo; finalmente, y no menos importante, señala que el registro de la documentación, incluida la resolución de emergencia, podrá ser posterior.

Tanto los oficios circulares como la resolución en nada modificaron la situación de emergencia que se vivía; básicamente fueron comunicados y actos administrativos que señalaban lo que en nuestra legislación ya disponíamos.

El plazo inicial de sesenta días, mediante decreto No. 1101, del 16 de junio de 2016, fue ampliado por treinta días más; luego, el 15 de julio -con decreto ejecutivo 1116- se expide un nuevo estado de excepción, esta vez únicamente para las provincias de Manabí y Esmeraldas, en el que se otorgan sesenta días. Motivados por las 
réplicas suscitadas el 10 de julio, este plazo fue prorrogado por treinta días -decreto 1191 del 13 de septiembre de 2016-.

Se declara una nueva emergencia -la tercera- con decreto 1215 de 14 de octubre, por sesenta días, a los cuales se sumaron treinta más mediante decreto 1274 del 13 de diciembre de 2016. El 12 de enero de 2017 -decreto 1295- se declara una vez más la excepcionalidad en las provincias de Esmeraldas y Manabí por sesenta días, y, al ser nuevamente renovada, se cumplió más de un año en estado de emergencia.

La primera emergencia la motivan los hechos que constan en los considerandos del decreto ejecutivo citado líneas antes de 17 de abril de 2016; la segunda, tercera, cuarta y demás con sus correspondientes extensiones son solicitadas mediante informes del Ministerio de Coordinación de Seguridad.

Es importante aclarar que el estado de excepción dictaminado por el Presidente de la República es el principal argumento de las administraciones centrales y seccionales para mantener la declaratoria de emergencia para las respectivas adquisiciones directas, no siendo un requisito sine equa non, empero, para el caso del terremoto se encontraron vinculados.

\section{BASE LEGAL NACIONAL PARA EL PROCEDIMIENTO DE EMERGENCIA}

En la Constitución de la República del Ecuador, en los artículos 164 y siguientes se establece que es potestad exclusiva del Presidente de la República decretar el estado de excepción, señalando que tendrá vigencia hasta un plazo máximo de sesenta días. Si las causas que lo motivaron persisten podrá renovarse hasta por treinta días más; ${ }^{4}$ este tipo de actos deben cumplir con los principios de: necesidad, proporcionalidad, legalidad, temporalidad, territorialidad y razonabilidad. ${ }^{5}$

Es importante mencionar que al final del artículo 166 de la referida norma se establece que "las servidoras y servidores públicos serán responsables por cualquier abuso que hubieran cometido en el ejercicio de sus facultades durante la vigencia del estado de excepción". ${ }^{6}$

Nuestra legislación contiene disposiciones para llevar a efecto procedimientos de contratación por emergencia, el artículo 6, numeral 31 de la Ley Orgánica del Sistema Nacional de Contratación Pública (LOSNCP) la define:

4. Ecuador, Constitución de la Republica, Registro Oficial No. 449 de 20-oct.-2008, art. 166.

5. Ibíd., art. 164.

6. Ibíd., art. 166 . 
Situaciones de Emergencia: son aquellas generadas por acontecimientos graves tales como accidentes, terremotos, inundaciones, sequías, grave conmoción interna, inminente agresión externa, guerra internacional, catástrofes naturales, y otras que provengan de fuerza mayor o caso fortuito, a nivel nacional, sectorial o institucional. Una situación de emergencia es concreta, inmediata, imprevista, probada y objetiva.

La situación acaecida el 16 de abril de 2016 se enmarca dentro de lo conceptualizado como emergencia, de manera expresa señala los terremotos, y enlista algunos condicionamientos:

a) Concreta: existe, es real, pero además es delimitada; para ello los datos con los que se comenzaron a escribir este artículo incluso permiten identificar lugares y consecuencias.

b) Inmediata: no espera, en este caso incluso se perdieron vidas.

c) Imprevista: no se puede conocer con anticipación para prevenir.

d) Probada: es demostrable, se pueda evidenciar.

e) Objetiva: se basa en hechos y no en percepciones.

El artículo 57 de la misma LOSNCP da cuenta del procedimiento de contratación en situación de emergencia:

a) La máxima autoridad de la entidad deberá emitir resolución motivada que declare la emergencia;

b) La entidad podrá contratar de manera directa, y bajo responsabilidad de la máxima autoridad, obras, bienes o servicios, incluidos los de consultoría, que se requieran de manera estricta para superar la situación de emergencia;

c) Se puede contratar empresas extranjeras sin requerir los requisitos previos de domiciliación ni presentación de garantías, los cuales se cumplirán una vez suscrito el respectivo contrato;

d) Superada la situación de emergencia, se publicará un informe que detalle las contrataciones realizadas y el presupuesto empleado.

En complemento con lo anterior, la codificación del SERCOP constante en la resolución No. INCOP No. 72-2016 de 31 de agosto de 2016, con sus respectivas reformas, establece en los artículos 361 y siguientes las condiciones para las contrataciones de emergencia que ratifican los puntos anteriores, además se enumera en detalle lo que se debe publicar una vez superada la emergencia con la particularidad de lo señalado en el artículo 364, respecto a que las publicaciones de las resoluciones de emergencia, los contratos y los informes, referidos en esta Codificación, se realizarán de manera inmediata a su emisión, otorgamiento o suscripción. 


\section{AnÁlisis de LOS PROCEDIMIENTOS DE EMERGENCIA}

Decretar una emergencia, y comenzar una delicada labor administrativa de llevar adelante procedimientos de contratación directa de por sí ya es complicado; si a esto le sumamos la destrucción y pérdida de vidas, desesperación y necesidades de la población, se vuelve aún más difícil.

Revisando varias de las emergencias publicadas en el portal «www.compraspublicas.gob.ec> se observa una serie de inconsistencias en las resoluciones publicadas. Así, más allá de polemizar, lo que realmente se pretende es analizar objetivamente cómo se debería proceder ante estas situaciones.

Como todo procedimiento de contratación lo primero que se necesita es identificar la necesidad a satisfacer, y es aquí que el contenido técnico tiene un importante aporte; los expertos en las distintas áreas: social, salud, infraestructura, vialidad, entre otras, son los encargados de definir el plan de acción respecto de hacia dónde deben apuntar los procedimientos de contratación. Por lo que podemos observar, en las zonas afectadas el ciudadano es el primer sujeto a considerar y a precautelar, para poder devolverle lo que tenía antes del desastre, no únicamente en el aspecto material, sino también restituir su entorno social y económico para que pueda retomar sus actividades diarias. Si bien las ayudas humanitaritas de los distintos niveles de gobierno no dejan de ser importantes, no es menos cierto que se debe apuntar a una solución integral económica, social y física para todos los damnificados.

Una vez identificadas las necesidades se debe proceder con inmediatez, pues no existe el tiempo que se tiene para los procedimientos convencionales: elaborar estudios previos, ni presupuesto referenciales ajustados a mercado. Sin embargo, eso no significa que no deba existir el análisis mínimo, la motivación necesaria y adecuada para atender inmediatamente con bienes, obras y servicios que permitan superar la emergencia en el menor tiempo posible; de esta forma se cumple con lo dispuesto en el artículo 23 de la LOSNCP, que establece que antes de iniciar un procedimiento precontractual la entidad deberá contar con los estudios.

La pregunta que surge es ¿cómo elaboro procedimientos de emergencia sin vulnerar las disposiciones legales vigentes? La respuesta no es fácil. Marco Morales menciona:

El obrar administrativo, no implica otra cosa que la ejecución de una norma dictada previamente y con toda certeza quien la confecciona, no siempre tendrá presente ni a la mano, todas las alternativas y vicisitudes que se generarán alrededor de la aplicación de determinada hipótesis jurídica, lo que provoca que, en un momento determinado, en el cual el ad- 
ministrador público deba optar por la resolución, se enfrentará a una serie de circunstancias de oportunidad y conveniencia. ${ }^{7}$

Se debe actuar rápidamente pero precautelando las actuaciones de la administración pública, si bien es cierto existe menos rigurosidad en las formas y autorizaciones como se observa en el artículo 57 de la LOSNCP, superada la situación de emergencia se publicará un informe que detalle las contrataciones realizadas y el presupuesto empleado. Es en esta instancia que deben ser documentadas motivadamente las actuaciones y la razón de ser de las decisiones apuradas que debió tomar la Administración, que no por ello debieron ser tomadas a la ligera o sin sustento.

No existen excepciones a lo señalado en el artículo 76 de la Constitución, letra 1) respecto a que:

Las resoluciones de los poderes públicos deberán ser motivadas. No habrá motivación si en la resolución no se enuncian las normas o principios jurídicos en que se funda y no se explica la pertinencia de su aplicación a los antecedentes de hecho. Los actos administrativos, resoluciones o fallos que no se encuentren debidamente motivados se considerarán nulos. Las servidoras o servidores responsables serán sancionados

La resolución que declara la emergencia debe tener sobra de argumentos y no únicamente referencias de disposiciones legales. Recordemos que motivación es la relación del hecho con el derecho: "la motivación es el juicio que forma la autoridad al apreciar el motivo y al ligarlo con la disposición de la ley aplicable, es decir la expresión de las razones que fundan y justifican el acto de la autoridad"; 8 por lo antes dicho, lo importante en la resolución que acoge llevar a cabo procedimientos de emergencia debe cumplir con este requisito. Ya lo dijo Patricio Secaira: la falta de motivación implica no solo vicio de forma sino también y principalmente vicio, arbitrariedad que como tal determina normalmente la nulidad del acto. ${ }^{9}$

Después de declarar la emergencia mediante acto administrativo, y haberla publicado lo antes posible en el portal de compras públicas, se procede a seleccionar al contratista; la misma norma establece que se lo puede hacer de manera directa, incluso puede ser un proveedor extranjero sin que sea necesario el cumplimiento de calificación en el Registro Único de Proveedores; es una facultad discrecional que se le otorga a la máxima autoridad. Legalmente se puede adjudicar a cualquiera; sin embargo,

7. Marco Morales Tobar, Manual de Derecho Administrativo (Quito: Corporación de Estudios y Publicaciones, 2001), 35 .

8. Gabino Fraga, Derecho Administrativo (México D. F.: Porrúa, 2009), 321.

9. Patricio Secaira, Curso Breve de Derecho Administrativo (Quito: Ed. Universitaria, 2004), 185. 
deben existir niveles mínimos de verificación económica, técnica y legal que permitan tener la certeza de que el oferente seleccionado va a estar en la capacidad de cumplir con el objeto por el cual se le requiere, y sobre todo dentro del menor tiempo posible.

La certificación presupuestaria es otro tema de discusión para estos casos. De manera imprescindible se ha de contar con los recursos necesarios para poder contratar, pues no hay excepción a lo dispuesto en el artículo 115 del Código Orgánico de Planificación y Finanzas Públicas respecto a que: "Ninguna entidad u organismo público podrán contraer compromisos, celebrar contratos, ni autorizar o contraer obligaciones, sin la emisión de la respectiva certificación presupuestaria".

Por otra parte, aunque no es requisito que las contrataciones por emergencia consten en el Plan Anual de Contratación, según el artículo 25 del Reglamento General a la Ley Orgánica del Sistema Nacional de Contratación Pública (RGLOSNCP), por cuanto no son previsibles, con lo que sí se tendrá que contar es con la certificación presupuestaria como requisito indiscutible. De manera previa se deberán realizar las modificaciones presupuestarias necesarias, ya sea que se cuenten con los recursos en los presupuestos de las entidades contratantes pero en distintas partidas, o, en su defecto, tengan que nutrirse de recursos mediante asignaciones, como se refleja en el artículo 3 del Decreto Ejecutivo 1001 y posteriores, que disponen al Ministerio de Finanzas que se sitúen los fondos necesarios para atender la situación de excepción, pudiendo utilizar las asignaciones presupuestarias disponibles, salvo las destinadas a salud y educación.

El SERCOP puso a disposición modelos de pliegos no obligatorios que faciliten la actuación administrativa de las entidades contratantes; dadas las circunstancias y por la emergencia real vivida, los pliegos se traducen a los requerimientos esenciales que la entidad contratante realiza. Al ser una contratación directa no existe un concurso de precios ni de mejores condiciones: queda a elección de la máxima autoridad escoger al contratista con las recomendaciones antes erigidas.

Surge en el debate si debe existir una resolución de adjudicación. La normativa aplicable no la exige debido a la inmediatez con la que deben realizarse las acciones en una emergencia real como la vivida en abril de 2016. Es evidente que no se dispone de todos los elementos que en un procedimiento convencional se requiere, empero existe la obligatoriedad de elaborar un contrato, y es a través de este instrumento que se deben plasmar todas y cada una de las condiciones que deben cumplir las partes.

Respecto a la solicitud de garantías dentro del procedimiento de contratación objeto de este análisis, el artículo 57 señala que para el caso de empresas extranjeras hay la posibilidad de no exigir garantías; sin embargo, establece que este requisito se cumplirá una vez suscrito el respectivo contrato, en otras palabras al final del día se deberían exigir garantías, las que fueran aplicables dados los condicionamientos de los artículos 74, 75 y 76 de la LOSNCP y como lo señala el artículo 69 de la misma 
ley, la cual prescribe: "Para la suscripción del contrato, será requisito previo la rendición de las garantías correspondientes".

Si bien es cierto que estos requisitos podrían demorar el inicio de los contratos, no es menos cierto que, como está escrita la norma, son requisito indispensable. En este análisis tratamos de no ser indolentes con la realidad de las personas afectadas por el terremoto, pero, al ser este un artículo de orden jurídico, lo que se intenta es transmitir un análisis objetivo desde el punto de vista práctico pero también legal del procedimiento de contratación por emergencia, para que se cumpla con el ordenamiento vigente.

El ya varias veces citado artículo 57 de la LOSNCP claramente señala que se contratará al amparo de la emergencia lo que "se requieran de manera estricta para superar la situación de emergencia", y es aquí que el buen criterio de autoridades y técnicos debe salir a la luz, ya que las compras deberán estar respaldadas con oportuna motivación para demostrar que lo contratado por emergencia sirve exclusivamente para superarla. Tenemos acertados ejemplos, como la demolición de edificaciones, provisión de vituallas, adquisición de kits de emergencia, readecuación de áreas destruidas, reparación de vías y alcantarillado, carpas y aditamentos para los albergues, entre otras, igual o mayormente esenciales.

Los procedimientos de emergencia no podrán ser utilizados como medio de elusión de procedimientos o empleados en objetos distintos que no sean los de superar la emergencia. Conocemos del argot popular la ya trillada frase: "es plata de todos porque lo pagamos con nuestros impuestos"; para este caso es aún más adecuada utilizarla por cuanto el pasado 20 de mayo de 2016 se expidió la Ley Orgánica de Solidaridad y de Corresponsabilidad Ciudadana para la Reconstrucción y Reactivación de las zonas afectadas por el terremoto de 16 de abril de 2016, cuyo objeto primordial es la recaudación de contribuciones solidarias con el propósito de permitir la planificación, construcción y reconstrucción de la infraestructura pública y privada, así como la reactivación productiva que comprenderá, entre otros objetivos, la implementación de planes, programas, acciones, incentivos y políticas públicas para enfrentar las consecuencias del terremoto.

Antes de realizar una contratación al amparo de esta contratación especial, se debe ser muy prolijo en la utilización de este régimen de contratación excepcional ya que su razón de ser es estrictamente la de superar la emergencia. Habrá que revisar la situación que existía antes del terremoto, la situación actual y la situación futura. Existe un dato que se mencionó al inicio que puede darnos un buen ejemplo: el 74\% de la población no contaba con servicios básicos. No sería prudente que, al amparo de la emergencia, se contrate la provisión de estos servicios si antes no existieron o no fue afectada estructura pública alguna que reparar o reemplazar. 
Respecto a las contrataciones realizadas, no se debió dejar de priorizar en la medida de lo posible la producción nacional y particularmente la producción local, más aún cuando mediante la reconstrucción la encomia popular y solidaria podía actuar como contratista y subcontratista, convirtiendo a los procedimientos de contratación por emergencia también en un elemento dinamizador de la economía.

Por lo dicho anteriormente, se disparan otras aristas respecto a quién y en dónde se deben aplicar los procedimientos de emergencia. La respuesta será siempre en los lugares en cuyas áreas sus competencias fueron afectadas por el terremoto. Existen organismos desconcentrados de carteras de Estado cuya matriz se encuentra en la capital, cada organismo gubernamental se encarga de solucionar lo que dentro de su jurisdicción le compete, pero para hacerlo armónicamente se deberá coordinar con los gobierno autónomos donde territorialmente se encuentren sus dependencias y a estas se suman las que cada municipio deberá llevar a cabo; para ello, mediante Decreto Ejecutivo No. 1004 de 26 de abril de 2016, se creó el Comité de Reconstrucción y Reactivación Productiva y del Empleo en las Zonas Afectadas por el Terremoto del 16 de abril de 2016 y cuyos objetivos primordiales son los de coordinar, priorizar y planificar los planes, proyectos y programas que permitan la reconstrucción y reactivación de las áreas devastadas por el terremoto.

\section{CONCLUSIONES}

El terremoto del 16 de abril permitió evidenciar que la Administración -refiriéndome a todos los niveles de gobierno-, a pesar de contar con disposiciones legales que definen y reglamentan las contrataciones por emergencia, al momento de llevarlas a cabo denotan importantes inconsistencias.

La inversión para la reconstrucción de Manabí y Esmeraldas supera los mil millones de dólares ${ }^{10}$ según la información proporcionada por el mismo Presidente de la República en uno de sus enlaces ciudadanos de finales del año pasado.

Mientras más papeleo exista dentro de una emergencia, más se puede dudar que haya sido real, ya que demuestra el tiempo y la oportunidad para generar un sin número de documentos.

10. "La inversión para la reconstrucción de Manabí y Esmeraldas llega a $\$ 1.000$ millones", diario El Telégrafo, 17 de diciembre de 2016. Disponible en 〈http://www.eltelegrafo.com.ec/noticias/politica/2/lainversion-para-reconstruir-manabi-y-esmeraldas-llega-a-usd-1-000-millones〉. Consulta: 6 de marzo de 2017. 
La pregunta que se deberían hacer autoridades antes de realizar una compra excepcional de emergencia es: ¿lo que se va a adquirir -bien, obra, servicio, consultoríapermite superar la emergencia? La respuesta será la que permita identificar si estamos cumpliendo con los presupuestos legales.

El éxito de la reconstrucción de las zonas afectadas por el sismo dependerá de forma importante de las adecuadas contrataciones por excepción realizadas para superar la emergencia, a lo que se suma la importante labor que realicen autoridades, administradores de contrato y contratistas de manera coordinada, sin quitar de vista en que el objetivo es devolverle el bienestar al ciudadano.

Los recursos que se utilicen para contratar los bienes, obras y servicios, incluidos los de consultoría, deben ser empleados con la responsabilidad que ello implica, en razón de que todos, a través de las distintas medidas tributarias adoptadas, estamos apoyando para la reconstrucción.

Es importante que los informes que se presenten para el cierre de las emergencias contengan argumentos y resultados que justifiquen y evidencien que el procedimiento de contratación especial empleado fue por la emergencia, y que se logró superarla sin dejar de cumplir disposiciones legales vigentes.

Son cuatro las emergencias decretadas por este evento natural que han provocado que permanezcamos casi un año en estado de excepción para las contrataciones, lo que provoca algunas dudas: ¿era necesario permanecer un año en estado de excepción?, ¿no es demasiado tiempo para haber realizado las contrataciones necesarias?, ¿se ha superado o no la emergencia? Con todos los informes preparatorios que se han elaborado, ¿no era más transparente llevar procedimientos de contratación convencionales que permitan la participación abierta de proveedores y no seguir contratando de manera directa?, ¿el legislador, al delimitar el plazo máximo de sesenta días y treinta máximo de renovación, no limita un tiempo prudencial? (lo que en este caso se ha utilizado por cuatro ocasiones).

Lo único cierto es que existirá una real reactivación y resarcimiento a los afectados cuando no solamente se hayan podido arreglar o reemplazar las edificaciones destruidas. Se verá superada la emergencia cuando los albergues hayan sido sustituidos por hogares, donativos por trabajo, subsidios por emprendimientos, y angustia por tranquilidad.

\section{BIBLIOGRAFÍA}

Fraga, Gabino. Derecho Administrativo. México D. F.: Porrúa, 2009.

"La inversión para la reconstrucción de Manabí y Esmeraldas llega a \$ 1.000 millones". Diario El Telégrafo, 17 de diciembre de 2016. Disponible en 〈http://www.eltelegrafo.com.ec/no- 
ticias/politica/2/la-inversion-para-reconstruir-manabi-y-esmeraldas-llega-a-usd-1-000-millones〉.

Morales, Marco. Manual de Derecho Administrativo. Quito: Corporación de Estudios y Publicaciones, 2001.

Secaira, Patricio. Curso Breve de Derecho Administrativo. Quito: Editorial Universitaria, 2004.

Secretaría de Gestión de Riesgos. Informe de situación No. 69. 19 de mayo de 2016. Disponible en http://www.gestionderiesgos.gob.ec/wp-content/uploads/downloads/2016/05/ Informe-de-Situaci\%C3\%B3n-69-\%E2\%80\%93-19052016-\%E2\%80\%93-12H30.pdf〉.

“6.622 viviendas y 71 kilómetros de vías fueron destruidas por el terremoto". El Telégrafo, 23 de abril de 2016. Disponible en 〈http:/www.eltelegrafo.com.ec/noticias/ecuador/3/6622-viviendas-fueron-destruidas-por-el-terremoto〉.

\section{Otros}

Ecuador. Código Orgánico de Planificación y Finanzas Públicas. Registro Oficial, Suplemento No. 306. Quito, 22 de junio de 2013.

Ecuador. Constitución de la República. Registro Oficial No. 449, de 20 de octubre de 2008.

Ecuador. Ley Orgánica del Sistema Nacional de Contratación Pública. Registro Oficial, Suplemento No. 395. Quito, 4 de agosto de 2008.

Ecuador. Presidencia de la República. Reglamento General a la Ley Orgánica del Sistema Nacional de Contratación Pública, Decreto Ejecutivo 1700. Registro Oficial, Suplemento No. 588. Quito, 12 de mayo de 2009.

Ecuador. Reglamento para el Control de la Discrecionalidad, Decreto Ejecutivo 3179, Registro Oficial 686. Quito, 18 de octubre de 2002. 